'Sección de Gastroenterología Hospital Clínico Universidad de Chile.

${ }^{2}$ Hospital Victoria IX Región. Clínica Dávila, Santiago. ${ }^{3}$ Laboratorio de Parasitología Básico-Clínico. Programa de Biología Celular y Molecular.

ICBM. Facultad de Medicina Universidad de Chile.

aAlumno de $7^{\circ}$ año de

Medicina. Facultad de Medicina. Universidad de Chile.

Recibido el 29 de marzo de 2014, aceptado el 26 de septiembre de 2014.

Correspondencia a: Dr. Luis Carlos Gil Sección de Gastroenterología, Hospital Clínico Universidad de Chile. Santos Dumont 999 Teléfono-fax: 229786122 drlcgill@yahoo.com

\section{Fascioliasis hepática humana: resistencia al tratamiento con triclabendazol}

\author{
LUIS CARLOS GIL ${ }^{1}$, ALEX DÍAZ ${ }^{1}$, CARLOS RUEDA ${ }^{2}$, \\ CHRISTIAN MARTÍNEZa, DOUGLAS CASTILLO ${ }^{3}$, WERNER APT $^{3}$
}

\section{Resistant human fasciolasis. Report of four patients}

Human fascioliasis is a parasitic zoonosis that affects the liver of human and herbivorous animals. In chronic cases, its diagnosis is confirmed by direct visualization of parasitic eggs in stool examination, by positive testing for Fasciola hepatica antigens in stools, or by direct observation of parasites by endoscopic retrograde cholangiography or surgery. In acute cases, serological reactions as immunoblothing or detection of parasite antigens in the blood are useful. The treatment of choice is triclabendazole. However, parasite resistance in animals, as well as in man, has been reported to this drug. We report four patients in whom the parasitic infection persisted despite a course of treatment with triclabendazole.

(Rev Med Chile 2014; 142: 1330-1333)

Key words: Cholangiography; Fasciola hepatica; Triclabendazole.
L a fascioliasis hepática es una zoonosis parasitaria causada por el trematodo, Fasciola hepatica, afecta al hígado del humano y animales herbívoros como vacunos, ovinos, caprinos $y$ equinos, entre otros ${ }^{1,2}$. El hombre se infecta, generalmente, por el consumo de berros y otros vegetales contaminados con metacercarias ${ }^{3}$ y en menor escala agua contaminada. En fase crónica de la infección, las formas adultas del parásito se localizan principalmente en las vías biliares del hígado de animales y del hombre ${ }^{4,5}$, siendo menos frecuente en vesícula biliar. Se manifiesta principalmente por dolor en el hipocondrio derecho, malestar general, fiebre e ictericia. Se sospecha el diagnóstico por la eosinofilia en el hemograma, alteraciones del perfil hepático y de la ecotomografía abdominal. La resonancia magnética (RM) y/o escáner abdominal (TAC) en fase aguda muestran hematomas subcapsulares y en fase crónica se puede apreciar nódulos, pseudotumores y defectos de llene compatibles con la silueta del parásito $^{6,7}$ (Figuras 1 y 2). El diagnóstico se confirma por hallazgo de huevos de F. hepatica en el examen copropasitológico (Figura 3) o extracción de parásitos adultos durante la cirugía biliar o colangiografía endoscópica retrograda (ERCP $)^{5}$. El tratamiento farmacológico de elección en las últimas décadas ha sido el triclabendazol (TBZ), altamente eficaz tanto en humanos como en animales ${ }^{8,9}$, dejando a un lado productos también eficaces, como la dehidroemetina y el bitionol, pero con mayores efectos colaterales en humanos. En Chile esta parasitosis es endémica en animales de abasto, principalmente en el sur del país, los casos en humanos también han sido reportados, pero sin la incidencia descrita en Perú y Bolivia ${ }^{8,10}$. En la década 1990-99 se disponía de TBZ para uso humano comercializado como Egaten ${ }^{\circledR}$, y para uso veterinario denominado Supolen ${ }^{\circledR}$; desde hace varios años no se dispone de la presentación para uso en humanos en Chile, por lo que algunos médicos han tratado a sus pacientes con formas farmacéuticas adaptadas a partir del TBZ de uso veterinario, con buenos resultados, al igual que en animales o con lotes de TBZ suministrados por la Organización Panamericana de Salud (OPS). La 

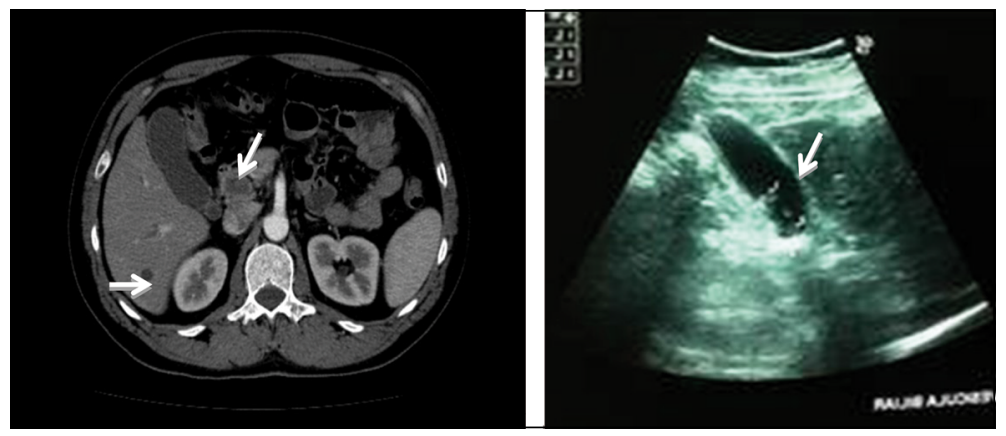

Figura 1. Caso 2. A: TAC de abdomen con dilatación de vía biliar y pequeño nódulo hepático; B: Ecotomografía abdominal con evidencia de fasciolas en la vesícula biliar.

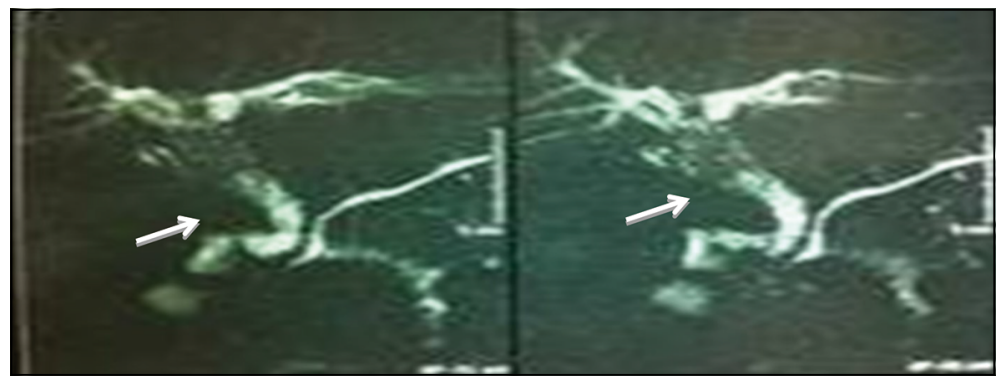

Figura 2. Caso 2. Colangiografía por resonancia magnética muestra conducto colédoco dilatado (flecha) por "enjambre o nido" de fasciolas.

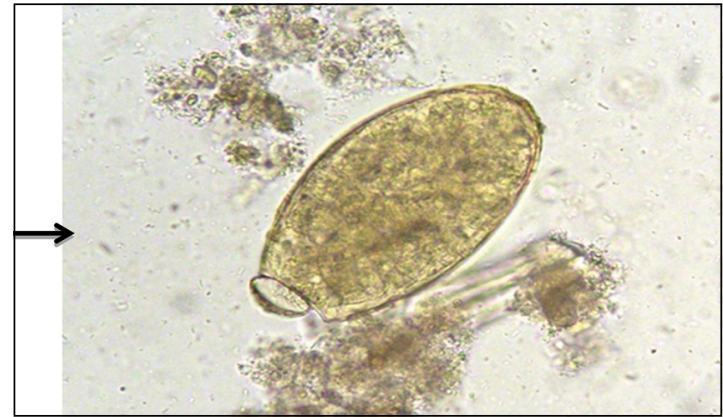

Figura 3. Caso 3. Huevo de Fasciola hepatica en coproparasitológico.

alta incidencia de esta parasitosis principalmente en vacunos y ovinos en el sur de Chile, y el uso de TBZ como droga de elección ${ }^{11}$, ha hecho que se describan casos de resistencia al fármaco en el viejo mundo y en América, en países vecinos como Argentina, Perú y Bolivia, inicialmente reportados en animales ${ }^{12-15}$, posteriormente y en menor proporción en humanos ${ }^{16,17}$. La resistencia al TBZ en Chile, a pesar de no existir grandes publicaciones, también ha sido descrita por los veterinarios en animales ${ }^{18}$. En este artículo se describen cuatro casos de humanos que fueron tratados en repetidas oportunidades con diferentes lotes de TBZ.

\section{Caso 1}

Hombre de 63 años, enfermero de profesión, consumidor de berros que en mayo de 2011 consultó por dolor abdominal en hipocondrio derecho, asociado a fiebre hasta $39^{\circ} \mathrm{C}$, diaforesis, decaimiento y malestar general. $\mathrm{Al}$ examen físico se evidenció dolor a la palpación en hipocondrio derecho. La ecotomografía abdominal sugirió presencia de $F$. hepatica en el colédoco y en el hemograma presentó recuento de 850 eosinófilos por $\mathrm{mm}^{3}$. El examen coproparasitológico seriado confirmó presencia de huevos de F. hepatica. Se trató con TBZ $20 \mathrm{mg} / \mathrm{kg}$ de peso. Evolucionó con mejoría de sus síntomas, y disminución de la eosinofilia hasta 150 por $\mathrm{mm}^{3}$. En octubre de 2012 consultó nuevamente por síntomas similares. En el hemograma esta vez había 2.800 eosinófilos por $\mathrm{mm}^{3}$ y el examen coproparasitológico nuevamente evidenció huevos de F. hepatica. El perfil hepático mostró un aumento de dos veces el valor normal para las transaminasas y de tres veces para las fosfatasas alcalinas. La RNM sugirió presencia del parásito en la vía biliar, por lo que se decidió realizar ERCP, extrayéndose tres ejemplares de $F$. hepatica del colédoco. Se reforzó el tratamiento con TBZ (Egaten ${ }^{\circledR}$ ) suministrado por la OPS, 20 
$\mathrm{mg} / \mathrm{kg}$ en dos dosis, con mejoría de los síntomas y disminución de eosinofilia a $250 \mathrm{~mm}^{3}$. En agosto de 2013 reaparecieron síntomas y en una nueva ERCP se extrajo dos ejemplares adultos de $F$. hepatica.

\section{Caso 2}

Hombre de 57 años que vive y trabaja en zona rural de Victoria, IX región. Realiza labores agrícolas y ganaderas. Niega ingesta de berros, pero consume agua en los pozos y esteros donde beben los animales. En septiembre de 2009 consultó por dolor en el hipocondrio derecho, asociado a fiebre hasta $39^{\circ} \mathrm{C}$. El recuento de eosinófilos en el hemograma era de 3.200 por $\mathrm{mm}^{3}$ y la TAC de abdomen mostró alteraciones sugerentes de infección por $F$. hepatica. Un test de ELISA resultó positivo para fascioliasis. Recibió tratamiento de $20 \mathrm{mg} / \mathrm{Kg}$ de peso con TBZ logrando estabilizar su sintomatología. En noviembre de 2011 repitió síntomas, con hallazgos similares en el hemograma y TAC abdominal. Una ecografía sugirió cálculos en vía biliar y colédoco. La ERCP permitió extraer cuatro ejemplares de F. hepatica. En la colecistectomía se evidenció tres ejemplares de F. hepatica en la vesícula. Recibió nuevo tratamiento con TBZ aliviando los síntomas y disminuyendo la eosinofilia. En abril de 2012 presentó síntomas similares. La RM de hígado y vía biliar sugirió presencia de parásitos en colédoco. ERCP permitió extraer cuatro ejemplares adultos de F. hepatica. El control post ERCP con RNM evidenció la persistencia de una imagen compatible con una agrupación de fasciolas en el conducto colédoco. Se administró nuevo tratamiento, con Egaten ${ }^{\circledR}$ suministrado por la OPS con mejoría de los síntomas. En diciembre de 2013 nueva ERCP confirmó la persistencia de la infección al extraerse dos ejemplares de F. hepatica.

\section{Caso 3}

Mujer de 45 años con antecedentes de consumo de berros. En agosto de 2012 consultó por dolor abdominal difuso. Presenta 2.400 eosinófilos por $\mathrm{mm}^{3}$ en el hemograma. La serología (ELISA) y el coproparasitario fueron positivos para $F$. hepatica. Recibió tratamiento con dosis total de $20 \mathrm{mg} / \mathrm{Kg}$ de peso de TBZ. Al control del mes siguiente presentó reactivación de los síntomas, persistencia de eosinofilia y presencia de huevos de F. hepatica en el examen coproparasitológico. Recibió nuevamente tratamiento con TBZ con leve remisión de síntomas. En diciembre de 2012 el hemograma y coproparasitológico fueron similares a los de agosto, recibiendo nuevamente TBZ (Egaten ${ }^{\circledR}$ suministrado por la OPS), con mejoría de sus síntomas. En diciembre de 2013 se efectúa un control mediante examen el coproparasitológico donde nuevamente evidenció huevos de $F$. hepatica (Figura 3).

\section{Caso 4}

Mujer de 54 años, ama de casa, refiere consumo de berros. En julio de 2011 consultó por dolor en hipocondrio derecho. Hemograma sin eosinofilia, bilirrubina total $1,3 \mathrm{mg} / \mathrm{dl}$, F. alcalina $380 \mathrm{UI} / \mathrm{L}$ (VN: hasta 170), GOT $170 \mathrm{UI} / \mathrm{L}$ (VN: hasta 73), GPT $180 \mathrm{UI} / \mathrm{L}$ (VN: hasta 70), Eco abdominal sugirió coledocolitiasis, ERCP permitió extraer un ejemplar adulto de F. hepatica. Se trató con TBZ (Egaten ${ }^{\circledR}$ ). En mayo de 2012 dolor abdominal, nueva eco abdominal sugirió presencia de $F$. hepatica. Nueva ERCP logró extraer tres ejemplares adultos de F. hepatica.

\section{Discusión}

La fascioliasis hepática es una zoonosis parasitaria endémica que existe en todo el mundo ${ }^{2}$. En Chile prevalece preferentemente en zonas del sur $^{1,4}$. El tratamiento de elección es el TBZ ${ }^{8,15}(\mathrm{La}-$ verde L. Efecto del nosodes fasciolinum y del fármaco triclabendazol sobre la oviposición de Fasciola hepatica en bovinos. Tesis Maestría Valdivia: Facultad de Ciencias Veterinarias, Univ. Austral de Chile 2007; 78 p.). Por no ser una parasitosis de alta prevalencia en el humano, la industria farmacéutica descontinuó la producción masiva de TBZ para uso humano, fabricando sólo lotes aislados y ocasionales para la OMS, OPS y con destino a países con mayor incidencia de esta zoonosis en humanos y animales de abasto (en América, Perú y Bolivia). En esta casuística, la infección se originó por el consumo de berros en 3 casos y en uno por consumo de agua de manantiales en donde solían beber vacunos y ovinos. La forma de presentación 
clínica de todos los pacientes estudiados es similar a la descrita en la literatura. Los 4 casos se trataron con TBZ y tienen en común que se documenta la persistencia de F. hepatica postratamiento, después de varios ciclos, además de tratamientos intervencionales como cirugía y ERCP. Todos los pacientes negaron consumo o contacto de fuentes potencialmente infectantes postratamiento que hicieran plantear una reinfección como causa de la persistencia de la parasitosis luego de un primer tratamiento farmacológico.

El antecedente de resistencia de la $F$. hepatica al TBZ tanto en humanos, como en animales, descrito en otras latitudes (en Chile sólo en animales), nos permiten plantear que estos casos corresponderían a la primera resistencia del parásito en seres humanos descrita en Chile. Estos pacientes están siendo sometidos a vigilancia periódica de comprobarse persistencia del parásito se plantea retratarlos con dehidroemetina y eventualmente nitaxozanida que no ha demostrado ser más eficiente que el TBZ o la dehidroemetina ${ }^{18,19}$.

\section{Referencias}

1. Apt W, Aguilera X, Vega F, Alcaíno H, Zulantay I, Apt P, et al. Prevalence of fascioliasis in humans, horses, pigs, and wild rabbits in 3 Chilean provinces. Bol Oficina Sanit Panam 1993; 115: 405-14.

2. Mas-Comas S. Fascioliasis. Capítulo 50. Parasitología Humana. Edit Werner Apt. Edit. Mc Graw Hill 2013; 379-89.

3. Fairweather I. Triclabendazole: new skills to unravel an old (ish) enigma. J Helminthol 2005; 79: 227-34.

4. Fairweather I. Triclabendazole progress report, 20052009: an advancement of learning? J Helminthol 2009; 83: 139-50.

5. Valenzuela G. [Epidemiological study on the development of Fasciola hepatica eggs in the environment of Valdivia, Chile (author's transl)]. Bol Chil Parasitol 1979; 34: 31-5.

6. Apt W, Zulantay I, Benavente R. Fasciolosis aguda: caso clínico. Parasitol Latinoam 2002; 57: 1-2.

7. Carrada-Bravo T. Fascioliasis: Diagnóstico, epidemiologia y tratamiento. Rev Gastroenterologia Mex 2003; 68: $135-42$.
8. Fairweather I, Boray JC. Fasciolicides: efficacy, actions, resistance and its management. Vet J 1999; 158: 81-112.

9. Díaz R. GM, Millan L, Pérez J, Millán JC. Comportamiento clínico-terapéutico de Fasciola hepatica en una serie de 87 pacientes. Rev Cubana Med Trop 2011; 63: 268-74.

10. Mamani W. CR. Determinación de resistencia antihelmíntica (Fasciola hepatica) en ovinos frente a Albendazol y Triclabendazol, La Paz-Bolivia. Rev Investig Perú 2009; 20: 254-62.

11. Millan M, Cárdenas A, Carrasco C. Parásitos de Fasciola hepatica intracoledociano. Rev Chil Cir 2008; 60: 332-5.

12. Moll L, Gaasenbeek CP, Vellema P, Borgsteede FH. Resistance of Fasciola hepatica against triclabendazole in cattle and sheep in The Netherland. Vet Parasitol 2000; 91: 153-8.

13. Olaechea F, Lovera V, Larroza M, Raffo F, Cabrera R. Resistance of Fasciola hepatica against triclabendazole in cattle in Patagonia (Argentina). Vet Parasitol 2011; 178: 364-6.

14. Ortiz P, Scarcella S, Cerna C, Rosales C, Cabrera M, Guzmán M, et al. Resistance of Fasciola hepatica against Triclabendazole in cattle in Cajamarca (Perú): a clinical trial and an in vivo efficacy test in sheep. Vet Parasitol 2013; 195: 118-21.

15. Cabada MM, White AC, Jr. New developments in epidemiology, diagnosis, and treatment of fascioliasis. Curr Opin Infect Dis 2012; 25: 518-22.

16. Keiser J, Utzinger J, Vennerstrom JL, Dong Y, Brennan G, Fairweather I. Activity of artemether and OZ78 against triclabendazole-resistant Fasciola hepatica. Trans R Soc Trop Med Hyg 2007; 101: 1219-22.

17. Mottier L, Álvarez L, Fairweather I, Lanusse C. Resistance-induced changes in triclabendazole transport in Fasciola hepatica: ivermectin reversal effect. J Parasitol 2006; 92: 1355-60.

18. Zumaqueo-Ríos JL, Sarracent-Pérez J, Rojas-García R, Rojas-Rivero L, Martínez-Tovilla Y, Valero MA, et al. Fascioliasis and intestinal parasitoses affecting schoolchildren in Atlixco, Puebla State, Mexico: epidemiology and treatment with nitazoxanide. PLoS Negl Trop Dis 2013; 7 (11): e2553.

19. Favennec L, Jave Ortiz J, Gargala G, López Chegne N, Ayoub A, Rossignol JF. Double-blind, randomized, placebo-controlled study of nitazoxanide in the treatment of fascioliasis in adults and children from northern Perú. Aliment Pharmacol Ther 2003; 17 (2): 265-70. 